\title{
MASTER
}

\section{Inductive Effects in Flux Conserving Tokamaks}

\author{
Glenn Bateman
}

\section{OAK RIDGE NATIONAL LABORATORY} OPERATED BY UNION CARBIDE CORPORATION - FOR THE DEPARTMENT OF ENERGY 


\section{DISCLAIMER}

This report was prepared as an account of work sponsored by an agency of the United States Government. Neither the United States Government nor any agency Thereof, nor any of their employees, makes any warranty, express or implied, or assumes any legal liability or responsibility for the accuracy, completeness, or usefulness of any information, apparatus, product, or process disclosed, or represents that its use would not infringe privately owned rights. Reference herein to any specific commercial product, process, or service by trade name, trademark, manufacturer, or otherwise does not necessarily constitute or imply its endorsement, recommendation, or favoring by the United States Government or any agency thereof. The views and opinions of authors expressed herein do not necessarily state or reflect those of the United States Government or any agency thereof. 


\section{DISCLAIMER}

Portions of this document may be illegible in electronic image products. Images are produced from the best available original document. 
Printed in the United States of America. Available from National Technical Information Service

U.S. Department of Commerce

5285 Port Royal Road, Springfield, Virginia 22161

Price: Printed Copy $\$ 4.50$; Microfiche $\$ 3.00$

This report was prepared as an account of work sponsored by an agency of the United States Government. Neither the United States Government nor any agency thereof, nor any of their employees, contractors, subcontractors, or their employees, makes any warranty, express or implied, nor assumes any legal liability or responsibility for any third party's use or the results of such use of any information, apparatus, product or process disclosed in this report, nor represents that its use by such third party would not infringe privately owned rights. 
ORNL / TM-6271

Dist. Category UC-20g

Contract No. W-7405-eng-26

FUSION ENERGY DIVISION

Inductive Effects in Flux

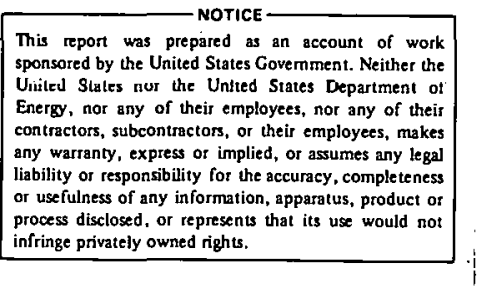

Conserving Tokamaks

Glenn Bateman

(Submitted to Physics of Fluids)

Date Published - June, 1978

Prepared by the

OAK RIDGE NATIONAL LABORATORY

Oak Ridge, Tennessee $\mathbf{3 7 8 3 0}$

operated by

UNION CARBIDE CORPORATION

for the

DEPARTMENT OF ENERGY 


\section{THIS PAGE \\ WAS INTENTIONALLY \\ LEFT BLANK}


CONTENTS

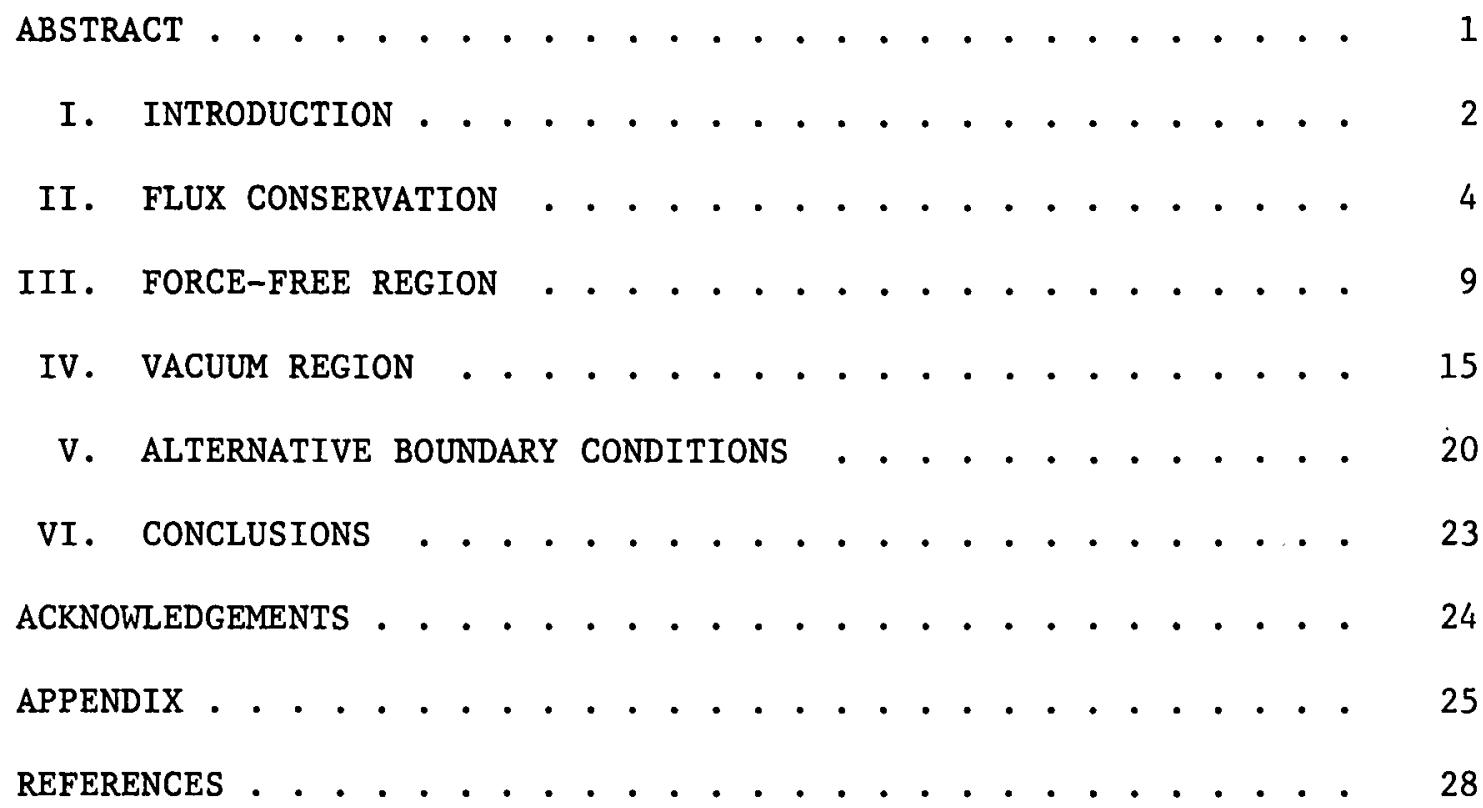


Scenarios are worked out in straight circular cylindrical geometry for the evolution of flux conserving sequences of equilibria as the plasma is heated or compressed. It is shown that force-free currents are induced when the low pressure region at the edge of the plasma is compressed against a flux conserving wall. A force-free surface current is induced if there is a vacuum region between the expanding plasma and the wall. These edge currents run opposite to the direction of the current in the main body of the plasma. The force-free currents can be avoided or reversed at the edge of a plasma with diffuse resistivity profile by programming the total longitudinal current as the plasma is heated or compressed. 


\section{INTRODUCTION}

There are essentially two ways to change a plasma equilibrium rapidly on the time scale of magnetic diffusion. The pressure profile can be changed by rapid heating, as in the proposed flux conserving tokamak experiments, ${ }^{1-4}$ or by sudden loss of confinement, as in the disruptive instability. ${ }^{5-7}$ Alternatively, the applied magnetic field can be increased, as in adiabatic compression experiments,,$^{8-12}$ or the shape can be changed, as in experiments which alter the plasma cross section. ${ }^{13-15}$ Plasma currents are induced as the equilibrium evolves in each case. Currents induced perpendicular to the magnetic field alter the confinement of plasma pressure. Currents induced parallel to the magnetic field have no direct effect on plasma pressure, but they can alter plasma stability. ${ }^{16,17}$ In particular, it has been reported ${ }^{17}$ that the appropriate profile of force-free current surrounding a toroidal plasma can increase the critical beta for stability against large-scale ballooning modes by an order of magnitude.

This paper is primarily concerned with the induction of currents parallel to the magnetic field at the edge of a hot plasma. Can substantial currents be driven in this edge region just by heating the central core of the plasma or by adjusting the externally applied magnetic fields in an experimentally reasonable way? How can we control the currents on a plasma-vacuum interface? Does most of the current decay or diffuse in a plasma with a continuous resistivity profile?

In order to elucidate the basic physics of these processes, the complications of toroidal geometry are set aside in most of this paper, and explicit examples are worked out in straight circular cylindrical 
geometry. ${ }^{18}$ Wherever possible, the effects of altering the geometry are pointed out. Most of the results depend only on Maxwell's equations and idealizations of the conductivity of the plasma, independent of the fluid model used. For some specific examples, the MHD equilibrium equation is used. 


\section{FLUX CONSERVATION}

$$
\text { Faraday's law }
$$

$$
\frac{\partial \mathrm{B}}{\partial \mathrm{t}}=-\nabla \times \underset{\sim}{\mathrm{E}}
$$

follows from the observation that the electric field around any closed contour is minus the rate of change of magnetic flux

$$
\psi=\int \mathrm{dS} \cdot \stackrel{\mathrm{B}}{\sim}
$$

through that contour. Using Galilean invariance as an approximation to relativistic invariance, Faraday's law is consistent with the transformation $^{19}$

$$
{\underset{\sim}{f i x e d}}_{f}=-v \times \underline{B}+\underbrace{}_{\text {moving }}
$$

where $\mathrm{v}$ is the relative velocity between the "fixed" and the "moving" frames of reference. The following mathematical identity can then be used to find the rate of change of flux through any moving contour

$$
\begin{aligned}
\frac{\mathrm{d}}{\mathrm{dt}} \psi & =\frac{\mathrm{d}}{\mathrm{dt}} \int \underset{\sim}{S} \cdot \underset{\sim}{\mathbb{B}} \\
& \equiv \int \mathrm{d} \underset{\sim}{S} \cdot\left[\frac{\partial \mathrm{B}}{\partial \mathrm{t}}+\underset{\sim}{\mathrm{v}} \underset{\sim}{\nabla} \underset{\sim}{\mathrm{B}}-\nabla \times(\underset{\sim}{\mathrm{v}} \times \underset{\sim}{\mathrm{B}})\right] \\
& =-\Phi \underset{\sim}{\mathrm{d} \ell} \cdot\left({\underset{\sim}{\mathrm{f}} \text { ixed }}_{\sim}^{\mathrm{v}} \times \underset{\sim}{\mathrm{B}}\right) .
\end{aligned}
$$


This identity can be used to show, for example, that fluxes are convected along with any conducting fluid

$$
\frac{\mathrm{d} \psi}{\mathrm{dt}} \mid \text { perfectly conducting fluid }=0 \text {, }
$$

since the electric field in the frame moving with the fluid is zero. If there are toroidal magnetic surfaces imbedded in the perfectly conducting fluid, it follows that the q-value

$$
\mathrm{q}(\psi) \equiv \mathrm{d} \psi_{\mathrm{tor}} / \mathrm{d} \psi_{\mathrm{pol}}
$$

is also convected along with the fluid

$$
\frac{\mathrm{dq}}{\mathrm{dt}} \mid \text { perfectly conducting fluid }=0 .
$$

In a resistive fluid, in the special case of flux surfaces with continuous symmetry (straight cylinder, axisymmetric toroid, or figure with helical symmetry), the rate of change of one flux relative to the other depends upon only the parallel electric field. To demonstrate this, consider the rate of change of the toroidal flux in the reference frame moving with the poloidal flux

$$
\begin{aligned}
& \frac{\mathrm{d} \psi_{\text {tor }}}{\mathrm{dt}}=-\Phi \underset{\sim}{\mathrm{d} \ell}:\left({\underset{\sim}{\psi_{\mathrm{pol}}}} \times \underset{\sim}{\mathrm{B}}+\underset{\sim}{\mathrm{E}}\right), \\
& \mathrm{C}_{\text {po1 }}
\end{aligned}
$$


where the electric field is measured in the frame of reference moving with the fluid. Applying the symmetry condition to Eq. (3), the component of poloidal flux velocity perpendicular to the flux surface. is

$$
v_{\psi_{\text {pol }}}=-E_{\text {tor }} / B_{\text {pol }}
$$

Then, using the poloidal components of the integrand in Eq. (8), it follows that

$$
\begin{aligned}
& \frac{\mathrm{d}}{\mathrm{dt}} \psi_{\text {tor }}=-\oint \mathrm{dl} \frac{\mathrm{E}_{\text {tor }}{ }^{\mathrm{B}_{\text {tor }}}+\mathrm{E}_{\mathrm{pol}}{ }^{\mathrm{B}_{p o 1}}}{\mathrm{~B}_{\mathrm{pol}}} \\
& C_{\text {pol }} \\
& =-\Phi \frac{\mathrm{d} l}{\mathrm{~B}_{\mathrm{pol}}} \underset{\sim}{\mathrm{F}} \cdot \underset{\sim}{\mathrm{R}} . \\
& c_{\text {pol }}
\end{aligned}
$$

This can be used to derive the relation

$$
\left.\frac{d y}{d t}\right|_{\psi}=-\frac{\partial}{\partial \psi}\left\langle v^{\prime}(\psi) \underset{\sim}{E} \cdot \underset{\sim}{B}\right\rangle
$$

where $\langle\ldots\rangle$ is a flux surface average. This was pointed out to me by D. J. Sigmar 20 and independently derived by R. N. Byrne and H. H. Klein. ${ }^{21}$ Two more equations will be needed in this paper. Ampere's law, with the displacement current neglected, is.:used to determine the current density 


$$
\underset{\sim}{J}=\nabla \times \underset{\sim}{\mathrm{B}} / \mu_{0}
$$

Any current perpendicular to the magnetic field exerts a force on the plasmà

$$
\rho \frac{\mathrm{dv}}{\mathrm{dt}}=-\nabla \mathrm{p}+\underset{\sim}{\mathrm{J}} \times \underset{\sim}{\mathrm{B}}
$$

where $p$ is a scalar pressure and $\rho$ is the mass density. The precise form of this equation of motion (e.g., the use of scalar pressure) is not essential for most of the results in this paper.

In order to illustrate how the motion of a perfectly conducting. fluid induces currents, consider the effect of plasma heating on the longitudinal magnetic field alone in a straight circular cylinder. As the central plasma pressure is raised, a force imbalance is produced, which drives an outward radial velocity. This velocity produces a poloidal $-v_{r} \times B_{z}$ electric field, as shown in Fig. 1. This reduces the longitudinal magnetic 'flux,: and therefore reduces the longitudinal magnetic field $B_{2}$ within the plasma according to Faraday's law. This change in the $B_{z}$ profile induces poloidal currents which provide an inward $\mathrm{J}_{\mathrm{e}} \times \mathrm{B}_{\mathrm{z}}$ force which then restores force balance at the higher plasma pressure. In the absence of viscosity, the plasma will overexpand and then oscillate around the new equilibrium.

If the plasma is surrounded by a flux conserving wall or be a set of high inductance toroidal field coils, as in most tokamaks, the toroidal magnetic field is compressed in the region around the expanding part of the plasma as in Fig. 2. This increase in the toroidal magnetic field around the plasma induces additional current in the toroidal 
field coils, which can be used to measure the diamagnetism of the plasma, and it can also induce force-free currents at the edge of the plasma. These force-free currents can have a stabilizing or a destabilizing effect on the plasma, depending upon their profile. This current profile can be altered by programming the poloidal field coil system. The force-free currents can be made to persist at the edge of the plasma in spite of resistivity as long as the plasma is heated and continues to expand or as long as the currents are driven by externally applied flux changes.

Consider, finally, the effect of plasma heating on the poloidal magnetic field alone. Once again, as the pressure is raised, it produces a force imbalance which drives an outward radial velocity. This time a longitudinal $-v_{\mathbf{r}} \times \mathrm{B}_{\theta}$ electric field is produced - everywhere in the same direction - which is largest at the edge of the plasma and zero in the center (see Fig. 3). From this electric field, Faraday's law predicts a decrease in the poloidal magnetic field, and hence a weaker inward $\mathrm{J}_{z} \times \mathrm{B}_{\theta}$ force is distributed over a larger area. Therefore, in the absence of a flux conserving poloidal field coil system, a strong toroidal magnetic field is needed to restore equilibrium force balance as the plasma pressure is increased. If the poloidal fiux expands in the center and compresses against a coil system at the edge, the longitudinal current will decrease in the center and increase at the edge, as detailed in the next two sections. 
III. FORCE-FREE REGION

A simple straight circular cylindrical model will be used in this section to demonstrate how force-free currents are induced when the low pressure edge of the plasma is squeezed against a flux conserving wall. In a straight circular cylinder, the fluxes, magnetic fields, current densities, and equilibrium pressure balance are given by

$$
\psi_{\text {tor }}(r)=2 \pi \int_{r}^{W} \mathrm{dr} r B_{z}(r)
$$

and

$$
\psi_{\text {pol }}(r)=L \int_{r}^{W} \mathrm{dr} B_{\theta}(r)
$$

where $L$ is the length of the cylinder under consideration and $w$ is the radius of the wall

$$
\begin{aligned}
& B_{z}(r)=-\frac{1}{2 \pi r} \frac{\partial}{\partial r} \psi_{t o r}(r) \\
& B_{\theta}(r)=-\frac{1}{L} \frac{\partial}{\partial r} \psi_{p o l}(r) \\
& J_{\theta}(r)=-\frac{1}{\mu_{0}} \frac{\partial}{\partial r} B_{z}(r) \\
& J_{z}(r)=\frac{1}{\mu_{0}} \frac{1}{r} \frac{\partial}{\partial r} r B_{\theta}(r) . \\
& \frac{\partial p}{\partial r}=J_{\theta} B_{z}-J_{z} B_{\theta} .
\end{aligned}
$$


An explicit analytic example of a flux conserving sequence of equilibria, as the pressure is raised in the central part of the plasma, is worked out in the Appendix. For a given expansion of the central core, we are concerned in this section with currents induced in an annulus of zero pressure but perfectly conducting plasma (low density but moderate temperature) extending to the wall. The pressure remains zero in the absence of heating in this region, since convection and compression do not produce pressure where before there was none.

As each element of the plasma is displaced by $\xi(r, t)$ in radius, let

$$
f(r, t)=r-\xi(r, t)=r_{o l d}\left(r_{n e w}\right)
$$

stand for the initial radius as a function of the new radius $r$ at time t. The boundary conditions for the low pressure annulus are

$$
f(w, t)=w
$$

and

$$
f\left(a_{t}, t\right)=a_{0}
$$

where $w$ is the radius of the wall and $a_{t}$ is the radius chosen for the edge of the hot central core which undergoes expansion from $a_{0}$ to $a_{t}$ in time $t$, as shown in Fig. 4. The property of flux conservation can then be written 


$$
\psi_{\text {tor }}(r, t)=\psi_{\text {pol }}(x)[f(r, t), 0]
$$

for both the toroidal and poloidal fluxes.

Assuming initially that there are no currents in the low pressure annulus

$$
\begin{aligned}
& \psi_{\text {tor }}(r, 0)=\pi B_{z w o}\left(w^{2}-r^{2}\right) \\
& \psi_{\text {pol }}(r, o)={ }^{2} B_{\theta w o} L \ln \dot{w} / r,
\end{aligned}
$$

where $\mathrm{B}_{\theta \text { wo }}$ and $\mathrm{B}_{z \text { wo }}$ are the initial $\mathrm{B}_{\theta}$ and $\mathrm{B}_{z}$ magnetic fields at the wall. From Eq. (24), the fluxes at time $t$ are

$$
\begin{aligned}
& \psi_{\text {tor }}(r, t)=\pi B_{z w o}\left[w^{2}-f^{2}(r, t)\right] \\
& \psi_{p o 1}(r, t)={ }^{w B}{ }_{\theta w o} L \ln [w / f(r, t)] .
\end{aligned}
$$

The magnetic fields and current densities calculated from these fluxes [Eqs. (16)-(19)] can then be used in the condition for zero pressure. equilibrium

$$
\mathrm{B}_{z} \mathrm{~J}_{\theta}=\mathrm{B}_{\theta} \mathrm{J}_{z}
$$

to derive an ordinary differential equation for $f(r, t)$ 


$$
-B_{z w o}^{2} \frac{f f^{-}}{r}\left(\frac{f f^{\prime}}{r}\right)^{\prime}=\left(w_{\theta w o}\right)^{2} \frac{f^{\prime}}{f} \frac{1}{r}\left(\frac{r f^{\prime}}{r}\right)^{\prime},
$$

where $f^{\prime} \equiv \partial f(r, t) / \partial r$, to be solved with boundary conditions of Eqs.

(22) and (23).

Under normal tokamak conditions,

$$
B_{\theta} / B_{z} \lll 1
$$

it is a simple matter to solve Eq. (30) iteratively. The lowest order approxination

$$
f^{2}=w^{2}+\left(r^{2}-w^{2}\right) \frac{w^{2}-a_{0}^{2}}{w^{2}-a_{t}^{2}}+O\left(\frac{B_{\theta}^{2}}{B_{z}^{2}}\right) w^{2}
$$

is adequate for our purposes. Substituting this expression back into Eqs. (16)-(19) and (27), we find that the toroidal magnetic field

$$
B_{z}(r, t)=B_{z w O}\left[\frac{w^{2}-a_{o}^{2}}{w^{2}-a_{t}^{2}}+O\left(\frac{B_{\theta}^{2}}{B_{z}^{2}}\right)\right]
$$

has been increased by the compression but remains uniform to leading order, since even a small poloidal current produces a large force. The poloidal magnetic field

$$
B_{\theta}(r, t) \simeq \frac{{ }^{r w B} \theta_{w o}}{r^{2}-w^{2} \frac{a_{t}^{2}-a_{0}^{2}}{w^{2}-a_{0}^{2}}}
$$


has also been increased, but it falls off more rapidly with radius than the original vacuum field. This implies a reversed longitudinal current density

$$
=\frac{-2 \frac{a_{t}^{2}-a_{o}^{2}}{w^{2}-a_{o}^{2}} w^{3} B_{\theta w o} / \mu_{0}}{\left(r^{2}-w^{2} \frac{a_{t}^{2}-a_{0}^{2}}{w^{2}-a_{0}^{2}}\right)^{2}}
$$

which is largest near the central core and remains finite out to the wall. The induction of this reversed force-free current is a geometrical effect; it does not appear in the corresponding model of a plane slab. The total longitudinal current in the annulus

$$
\begin{aligned}
I_{z \text { annulus }} & =\int_{a_{t}}^{w} d r r J_{z}=\left.\frac{r B_{\theta}}{\mu_{0}}\right|_{a_{t}} ^{w} \\
& =-\frac{w^{2}-a_{0}^{2}}{w^{2}-a_{t}^{2}}\left(\frac{a_{t}^{2}}{a_{0}^{2}}-1\right){ }^{w B_{\theta w o}} / \mu_{0}
\end{aligned}
$$

can be comparable to the longitudinal current in the plasma

$$
\begin{aligned}
I_{z \text { plasma }} & =\int_{0}^{a_{t}} d r \quad r J_{z}=\left.\frac{r B_{\theta}}{\mu_{0}}\right|_{0} ^{a_{t}} \\
& =\frac{w^{2}-a_{0}^{2}}{w^{2}-a_{t}^{2}} \frac{a_{t}^{2}}{a_{0}^{2}}{ }^{w B_{\theta w o}} / \mu_{0}
\end{aligned}
$$


only when there is a moderately large expansion of the central core on a flux conserving time scale. With diffusion, and with a continuously driven source and sink of heat, it should be possible to maintain these induced currents as illustrated in Fig. 5.

A simple estimate can be made for the amount of expansion produced by raising beta if we use the force balance equation written in the form

$$
B_{z}^{2}(r, t)=B_{z w t}^{2}-2 \mu_{o} p(r, t)+\int_{r}^{w} d r\left(\frac{\partial}{\partial r} B_{\theta}^{2}+2 B_{\theta}^{2} / r\right)
$$

and calculate the conservation of toroidal flux within the plasma with the approximation $\mathrm{B}_{2 \mathrm{~W}}^{2} \gg 2 \mu_{\mathrm{o}} \mathrm{p}(\mathrm{r})$. The result is

$$
\frac{a_{t}^{2}}{a_{0}^{2}} \simeq \frac{B_{z w o}}{B_{z w t}} \frac{1-\beta_{o}}{1-\beta_{t}}
$$

where

$$
\beta_{t}=\left(1-\frac{1}{\beta_{I}}\right) 2 \int_{0}^{a} t d r r p(r, t) / a_{t}^{2} B_{z w t}^{2} / 2 \mu_{0} \text {, }
$$

and the $\beta_{I}$ term lumps together the effects of confinement by the pololdal magnetic field, which are not important here. Equation (39) together with Eq. (33) y1elds

$$
B_{\geqslant w t .}=B_{z w n}\left(1+\frac{a_{0}^{2}}{w^{2}} \frac{\beta_{t}-\beta_{0}}{1-\beta_{t}}\right) .
$$


It can be seen that the amount of expansion is comparable to the magnitude of beta compared to unity.

In a previous study of plasma heating and flux conserving equilibria, Dory and Peng ${ }^{4}$ did not specifically consider the compression of flux against a fixed wall or a set of high inductance coils. They were able to avoid force-free currents by prescribing an increase in the longitudinal field $B_{z w t}$ at the wall or an expansion of the wall and plasma together.

IV. VACUUM REGION

Consider now the heating and expansion of a plasma surrounded by a vacuum or a highly resistive region, which in turn is surrounded by a perfectly conducting wall or a set of high inductance coils. Let $b_{t}$ be the radius of the plasma-vacuum interface with initial value $b_{0}$. Consider first the effect of plasma expansion without regard to any particular features of the plasma profile; assume only that $p\left(a_{t}, t\right)=0$ and $\partial \mathrm{p} /\left.\partial \mathrm{r}\right|_{\mathrm{r}=\mathrm{a}_{t}}=0$ at the edge of the plasma so that no discontinuities in pressure or its derivative ever develop there. These conditions immediately lead to a paradox that can be demonstrated in a very simple way.

Conservation of the q-value during the flux conserving motion of the edge of the plasma

$$
q\left(b_{t}, t\right)=q\left(b_{0}, 0\right)
$$


in straight circular cylindrical geometry implies

$$
\frac{\mathrm{B}_{\theta b t}}{\mathrm{~B}_{\theta b o}}=\frac{\mathrm{b}_{t}}{\mathrm{~b}_{\mathrm{o}}} \frac{\mathrm{B}_{z b t}}{\mathrm{~B}_{z \mathrm{bo}}} .
$$

This, together with the conservation of both total fluxes in the vacuum region,

$$
\begin{aligned}
& \psi_{\text {pol }}(t=0)=L b_{o} B_{\theta b o} \ln w / b_{0} \\
& \text { vac } \\
& =\psi_{p o 1}^{\prime}(t)=L b_{t}^{B} \theta b t \ln w / b_{t} \\
& \text { vac } \\
& \psi_{\text {tor }}(t=0)=\pi B_{z b o}\left(w^{2}-b_{o}^{2}\right) \\
& =\psi_{\operatorname{tar}}(t)=\pi B_{z b t}\left(w^{2}-b_{t}^{2}\right)
\end{aligned}
$$

yields three equations for the three unknowns $B_{\theta b t}, B_{z b t}$, and $b_{t}$

$$
\frac{B_{\theta b t}}{B_{\theta b o}}=\frac{b_{t} B_{z b t}}{h_{0} B_{z b o}}=\frac{b_{t}\left(w^{2}-b_{o}^{2}\right)}{h_{0}\left(w^{2}-b_{t}^{2}\right)}=\frac{b_{0} \ln w / b_{0}}{b_{t} \ln w / b_{t}}
$$

where. $B_{\theta b t}$ and $B_{z b t}$ are the poloidal and longitudinal magnetic fields at the plasma vacuum interface, with initial values $B_{\theta b o}$ and $B_{z b o}$. In the last of these three equations, where $b_{t}$ is the only unknown, 
a transcendental function of $b_{t}$ is equated to an algebraic function of $b_{t}$. The only solution to this equation is $b_{t}=b_{0}$, which implies $\mathrm{B}_{\theta b t}=\mathrm{B}_{\theta b o}$ and $\mathrm{B}_{z b t}=\mathrm{B}_{z b o}$. Under these conditions, it follows that there can be no induced diamagnetism within the plasma, since diamagnetism would require either an increase in $B_{z}$ at the edge of the plasma or an expansion of the plasma $\left(b_{t}=b_{0}\right)$ in order to conserve toroidal flux within the plasma. Therefore, under these conditions, the plasma pressure cannot be raised in a completely flux conserving way.

This simple argument demonstrates that a completely flux conserving motion is not possible in a circular cylindrical plasma surrounded by a vacuum and a flux conserving wall.

A resolution of this paradox was suggested by $D$. Pfirsch. ${ }^{2}$ A thin boundary layer of force-free current forms at the edge of the plasma, and the fluxes are not conserved locally within this boundary layer. In the limit of an arbitrarily thin boundary layer, the force-free surface current produces a discontinuity in the direction, but not in the magnitude, of the magnetic field. There is a discontinuity in the derivative, but not in the magnitude of the fluxes, at the plasma vacuum interface. Hence, there is a discontinuity in the q-value at this boundary, and Eqs. (42) and (43) are not appropriate. Lack of flux conservation at the plasma boundary is physically reasonable, since an infinitely resistive vacuum region must be separated from a zero resistance plasma by a highly resistive boundary layer. The more realistic diffuse resistivity profile will be considered in the next section of this paper. 
The induced force-free surface current can be determined in the following way. Let the magnetic fields just inside the plasma, $B_{\theta b t-}$ and $\mathrm{B}_{\mathrm{zbt-}}$, be determined by the conservation of the q-value there

$$
\frac{{ }^{B} \theta_{b t-}}{{ }^{B_{b o}}}=\frac{{ }_{t}}{b_{0}} \frac{B_{z b t-}}{B_{z b o}} \text {, }
$$

while the magnetic fields just outside the plasma, $B_{\theta b t+}$ and B ${ }_{z b t+}$, be determined by conservation of the fluxes in the vacuum region

$$
\begin{aligned}
& B_{z b t+}=B_{z b o} \frac{w^{2}-b_{o}^{2}}{w^{2}-b_{t}^{2}} \\
& B_{\theta b t+}=B_{\theta b o} \frac{b_{o} \ln w / b_{o}}{b_{t} \ln w / b_{t}} .
\end{aligned}
$$

A relation between the fields inside and outside the plasma follows from the requirement that a force-free surface current produces only a discontinuity in the direction of the field and not in its magnitude. This can be derived from Eq. (20).

$$
\mathrm{B}_{\mathrm{zbt}+}^{2}+\mathrm{B}_{\theta \mathrm{bt}+}^{2}=\mathrm{B}_{\mathrm{zbt}-}^{2}+\mathrm{B}_{\theta \mathrm{bt}-}^{2}
$$

This completes the set of equations for the magnetic fields at the surface of the plasma (given an expansion of the plasma $b_{t}$ ) driven by an increase in the plasma pressure. The solution for $\mathrm{B}_{\theta b t-}$ is 


$$
B_{\theta b t-}=B_{\theta b o} \frac{b_{t}}{b_{0}} \frac{{ }_{z b t t}}{B_{z b o}}\left[\frac{i+B_{\theta b t+}^{2} / B_{z b t+}^{2}}{1+\left(b_{t} B_{\theta b o} / b_{o} \dot{B}_{z b o}\right)^{2}}\right]^{1 / 2}
$$

or, in the large aspect ratio limit $\mathrm{B}_{\theta b o} \ll \mathrm{B}_{z b o}$,

$$
B_{\theta b t-} \simeq B_{\theta b o} \frac{b_{t}}{b_{0}} \frac{w^{2}-b_{t}^{2}}{w^{2}-b_{o}^{2}} .
$$

The magnitude of the force-free surface current is

$$
\begin{aligned}
I_{z b t} & =b_{t}\left(B_{\theta b t+}-B_{\theta b t-}\right) / \mu_{0} \\
& \simeq-I_{\text {plasma }}(t) \\
& {\left[1-\frac{\ln w / b_{t}}{\ln w / b_{o}} \frac{\left(w / b_{o}\right)^{2}-1}{\left(w / b_{t}\right)^{2}-1}\right] }
\end{aligned}
$$

As the plasma expands, this. force-free current is driven opposite to the direction of the main plasma current, and can be quite sizeable for moderate plasma expansion. This surface current can be avoided by holding the toroidal plasma current fixed as the plasma is heated and expands (a prescription applicable to circular cylindrical geometry) or by increasing the toroidal magnetic field to prevent plasma expansion (holding $b_{t}$ fixed) as the plasma is heated. These conditions will be studied in more detail in the next section on plasmas with continuous resistivity profiles. 
V. ALTERNATIVE BOUNDARY CONDITIONS

Up to this point we have considered only rigid flux conserving boundaries on which the parallel electric field is zero. In a resistive plasma, however, there is a positive longitudinal electric field impressed along the edge of the plasma. Here, positive means in the same sense as the current in the main body of the plasma. Then by Ohm's law

$$
-V_{r} B_{\theta}+\eta J_{z}=E_{z}
$$

it follows that there must be either a positive current density at the edge of the plasma or an inward plasma velocity there. It will be shown in this section that this applied electric field and inward plasma velocity make a positive contribution to the current at the edge of the plasma. This current can reduce or reverse the negative force-free current described in the last two sections. Programming the total longitudinal plasma current to be fixed in fact reduces the force-free current at the edge to zero in a cylindrical plasma.

In order to keep the edge of the plasma force free, the moving plasma must compress in such a way as to keep the longitudinal magnetic field $B_{z}$ uniform and the poloidal current $J_{0}$ zero to leading order in $B_{\theta} / B_{z}$. Then it follows from the longitudinal component of Faraday's law

$$
\frac{\partial B_{z}(t)}{\partial t}=-B_{z}(t) \frac{1}{r} \frac{\partial}{\partial r}\left[r v_{r}(r, t)\right]
$$


that the radial velocity in the force-free region must be

$$
v_{r}=\frac{w^{2}-r^{2}}{2 r} \frac{\dot{B}_{z}(t)}{B_{z}(t)}+v_{r w}(t) w / r
$$

to leading order in $B_{\theta} / B_{z}$, where $w$ is the radius of the wall and $v_{r w}(t)$ is the plasma velocity at the wall. It is assumed that new. gas becomes instantly ionized on the time scale of the motion as the plasma moves in off the wall. The terms in $v_{r}(r, t)$ that vary like $1 / \mathrm{r}$ are incompressible contributions which have no effect on $B_{z}(t)$, while the terms that vary like $r$ correspond to a uniform compression of the plasma and the $\mathrm{B}_{z}$ field.

Now consider the poloidal component of Faraday's law

$$
\frac{\partial B_{\theta}}{\partial t}=-\frac{\partial}{\partial r}\left(v_{r} B-\eta J_{z}\right)
$$

with $v_{r}(r, t)$ given by Eq. (56). If $n J_{z}$ is neglected, as the first step in an iterative solution, the rest of the equation is satisfied by Llıe fullowing similarity solution

$$
B_{\theta}(r, t)=\frac{{ }^{w B} \theta_{w o} r}{r^{2}-C(t)}
$$

where the function $c(t)$ is determined by the differential equation

$$
\frac{d}{d t}\left\{B_{z}(t)\left[C(t)-w^{2}\right]\right\}=2 v_{r w}(t) w_{z}(t)
$$


and hence

$$
C(t)=w^{2}\left[1-B_{z}(0) / B_{z}(t)\right]+\frac{2 w}{B_{z}(t)} \int_{0}^{t} d t^{\prime} v_{r w}\left(t^{\prime}\right) B_{z}\left(t^{\prime}\right)
$$

If the velocity at the wall $v_{\mathrm{rw}}(t)$ is zero, the resulting similarity solution for $B_{\theta}(r, t)$ in the force-free region agrees with Eqs. (34) and (33). An inward radial velocity at the wall has the effect of reducing $C(t)$.

From Eqs. (58) and (19), the longitudinal current density in the force-free region is

$$
J_{z}(\dot{r}, t)=\frac{-2 w B}{\theta_{w 0} C(t)} \frac{\mu_{0}\left[r^{2}-c(t)\right]^{2}}{.}
$$

A negative value for $c(t)$ is needed to induce a positive force-free current by compression under these conditions. According to Eq. (54), the inward velocity needed for this negative value of $g(t)$ would bo driven by a sufficlently large applied electric field as the edge of the plasma is compressed.

In most tokamaks, the external circuitry is arranged to hold the total longitudinal current fixed

$$
I_{z}(t)=\frac{2 \pi w}{\mu_{o}} B_{\theta_{w}}(t)=I_{z O} .
$$


Here, this implies $B_{\theta w}(t)=B_{\theta_{w o}}$ and hence $C(t)=0$ and

$$
v_{r w}(t)=-w \dot{B}_{z}(t) / 2 B_{z}(t)
$$

Under these conditions no force-free current, $J_{z}(r, t)=0$, is driven at the edge of the plasma. A programmed rise in the total longitudinal current on a flux conserving time scale would therefore be needed to drive the positive force-free current required to enhance the predicted stability of large-scale ballooning modes at high beta. From Eqs. and (62), programming the longitudinal current

$$
I_{z}(t)=\frac{I_{z}(t=0)}{1-c(t) / w^{2}}
$$

determines $C(t)$. This in turn determines the force-free current density [Eq. (61)] near the edge of the plasma.

VI. CONCLUSIONS

In this paper the plasma is considered in two parts - a high pressure central core surrounded by a relatively low pressure region near the edge. An example of a flux conserving sequence of equilibria for the high pressure core is worked out in the Appendix. It is shown that the low-pressure region near the edge of the plasma must compress uniformly [Eq. (56)] in order not to induce poloidal currents to lowest order in $\mathrm{B}_{\mathrm{pol}} / \mathrm{B}_{\text {tor }}$. A force-free current is induced in the direction opposite to that of the current in the main body of the plasma 
[Eqs. (35) and (53)] if this low pressure region is compressed against a fixed flux conserving wall. This force-free current can be avoided or reversed by programming the toroidal current (so that the wall no longer conserves $f(u x)$ as described in Sect. V. Work is under way to extend these findings to toroidal geometry.

\section{ACKNOWLEDGEMENTS}

I would like to thank D. Pfirsch, D. J. Sigmar, D. B. Nelson, Y-K. M. Peng, and A. T. Mense for useful discussions and contributions. Research sponsored by the Office of Fusion Energy (ETM), U.S. Department of Energy under contract W-7405-eng-26 with the Union Carbide Corporation. 
APPENDIX: SPECIFIC EXAMPLE OF A FLUX CONSERVING SEQUENCE OF EQUILIBRIA It is easy to construct analytic examples of flux conserving sequences of equilibria in straight circular cylindrical geometry. Consider here the hot central core of the plasma. Suppose initially

$$
B_{z}(r, 0)=B_{z O}
$$

and

$$
J_{z}=J_{z o}\left(1-r^{2} / a_{0}^{2}\right)^{\nu}=J_{z 0} x_{0}^{\nu}
$$

where $x_{0}=1-r^{2} / a_{0}^{2}$ and $\nu \geq 0$. Then the toroidal flux and $q$-value are given by

$$
\begin{aligned}
\psi_{\text {tor }}(r, 0) & =B_{z o} \pi a_{0}^{2}\left(1-x_{0}\right) \\
q(r, 0) & =\frac{4 \pi(\nu+1)}{\mu_{0} J_{z o} L} \frac{r^{2}}{a_{0}^{2}} \frac{B_{z o}}{1-\left(x_{0}\right)(\nu+1)} \\
& =\frac{4 \pi(\nu+1)}{\mu_{0} J_{z o}^{L}} \frac{\psi_{\text {tor }}}{\pi_{0}^{2}} \frac{1}{1-\left(1-\psi_{\text {tor }} / \pi a_{0}^{2} B_{z o}\right)^{\nu+1}},
\end{aligned}
$$

where $\mathrm{L}$ is the length of the cylinder under consideration. Now change the equilibrium by making $B_{z}(r, t)$ diamagnetic and allowing the plasma to expand to radius $a_{t}$, where $t$ is just an identifying parameter. Choose for example

$$
B_{z}(r, t)=B_{z t}\left(1-2 \delta X_{t}\right)
$$


where $x_{t}=1-r^{2} / a_{t}^{2}$, and $B_{z t}$ and $\delta$ are adjustable parameters. Conservation of toroidal flux

$$
\psi_{\text {tor }}(r, t)=B_{\bar{z} t} \pi a_{t}^{2}\left(1-\delta-x_{t}+\delta x_{t}^{2}\right)
$$

at the edge of the plasma implies

$$
a_{t}^{2} B_{z t}(1-\delta)=a_{0}^{2} B_{z o}
$$

This relation determines $\delta$, for example, while the parameters $B_{z t}$ and $a_{t}$ are determined by the boundary conditions as described in this paper.

Flux conservation requires that the function $q(\psi)$ remains unchanged. Then from Eqs. (A4) and (A6)

$$
q(r, t)=\frac{4 \pi(\nu+1)}{\mu_{0} J_{2 U} L} \frac{B_{z o}}{1-\delta} \frac{\left(1-\delta-K_{t} \cdot \delta X_{t}^{2}\right)}{1-\left(\frac{X_{t}-\delta X_{t}^{2}}{1-\delta}\right) \nu+1}
$$

This equation can be used to determine

$$
B_{\theta}(r, t)=\frac{2 \pi r B_{z}(r, t)}{L q(r, t)}
$$

from which $J_{\varepsilon}(r, t), J_{\theta}(r, t), \partial p / \partial r$, and hence $p(r, t)$ can all be determined.

In straight circular cylindrical geometry, it is much easier to calculate such sequences of flux conserving equilibria by giving the function $B_{z}(r, t)$ with a few adjustable parameters and then solving ultimately for $p(r, t)$, rather than the other way around. of course, any reasonable choice could have been made for $B_{z}(r, t)$ and for $q(r, 0)=$ $q\left[\psi_{\text {tor }}(r, t)\right]$ 


\section{REFERENCES}

${ }^{1}$ V. S. Mukhovatov and V. D. Shafranov, Nuc1. Fusion 11, 605-633 (1971), see in particular 6:30-631.

${ }^{2}$ J. F. Clarke; "Hi.gh Beta.Flux-Conserving Tokamaks," ORNL/TM-5429, Oak Ridge National Laboratory, Oak Ridge, Tennessee (1976).

3.J. F: Clarke and D. J. Sigmar, Phys. Rev. Lett. 38, 70-74 (1977).

${ }^{4}$ R. A. Dory and $Y-K$. M. Peng, Nucl. Fusion 17, 21-31 (1977).

${ }^{5}$ F. Karger et al., In Plasma Physics and Controlled Nuclear Fusion Research - 1976 (IAEA, Vienna, 1977), Vol. 1, 267-275.

${ }^{6}$ Equipe TFR, Nucl. Fusion 17, 1283-1296 (1977).

${ }^{7}$ I. H. Hutchinson, Phys. Rev. Lett. 37, 338-341 (1976).

${ }^{8}$ H. P. Furth and S. Yoshikawa, Phys. Fluids $\underline{13}, 2593-2596$ (1970).

${ }^{9} \mathrm{~K}$. Bol et al., Phys Rev. Lett. 29, 1495-1498 (1972).

${ }^{10}$ R. A. E1lis, Jr., in Proc. 3rd Symposium on Plasma Heating in Toroidal Devices (Varema, Tlaly, 1976), 173-184

${ }^{11}$ Y. N. Dnestrovskii, D. P. Kostomarov, and N. L. Pavlova, Zh Eksp. Teor. Fiz. Pis'ma Red. [Sov. Phys. - JETP Lett. 13, 493-495 (1971)].

${ }^{12}$ M. G. Kaganskij, S. G. Kalmykov, S. V. Lẻbedev, and K. G. Shakhovets, in Plasma Physics and Controlled Nuclear Fusion Research - 1976 (IAEA, Vienna, 1977), Vo1. 1, 387-397.

${ }^{13}$ G. Cima, D. C. Robinson, D. L1. Thomas, and A. J. Wootton, in Plasma Physics and Controlled Nuclear Fusion Research - 1976 (IAEA, Vienna, 1977), Vol. $1,335-350$.

${ }^{14}$ H. Grad, P. N. Hu, D. C. Stevens, and E. Turkel, in Plasma Physics and Controlled Nuclear Fusion Research - 1976 (IAEA, Vienna, 1977), Vol. $2,355-364$. 
${ }^{15} \mathrm{H}$. Grad, P. Hu, D. Stevens, Proc. Natl. Acad. Sci., USA $\underline{72}, 3789$ (1975).

${ }^{16}$ Y. N. Dnestrovskii, D. P. Kostomarov, and A. M. Popov, Zh. Tekh. Fiz. [Sov. Phys. - Tech. Phys. 17, 1463-1468 (1973)].

${ }^{17}$ D. A. D'Ippolito, J. P. Freidberg, J. P. Goedbloed, and J. Rem, in Plasma Physics and Controlled Nuclear Fusion Research - 1976 (IAEA, Vienna, 1977), Vo1. 1, 523-535; Jutphaas Report IR 77/036 (1977).

${ }^{18}$ D. J. Sigmar and G. Vahala, "Analytic High $B$, Flux Conserving Equilibria For Cylindrical 'lokamaks," ORNL/IM-6040, Oak Ridge National Laboratory, Oak Ridge, Tennessee (1978).

${ }^{19} \mathrm{~J}$. D. Jackson, in Classical Electrodynamics (John Wiley and Sons, New Iuik, 1962).

${ }^{20}$ D. J. Sigmar, in Proc. 8th European Conf. on Controlied Fusion and Plasma Phys. (Prague, 1977) 1, 70.

${ }^{21}$ R. N. Byrne and H. H. Klein, J. Comput. Phys., to be published.

${ }^{22}$ D. Pfirsch (Max-Planck Institut für. Plasma Physik), private communication, July 1977 . 
FIGURE CAPTIONS

Fig. 1. Raising the plasma pressure creates a force imbalance inducing an outward radial velocity and a poloidal $v_{r} \times B_{t o r}$ that results in a decrease of $\mathrm{B}_{z}$ within the plasma and an inward $\mathrm{J}_{\mathrm{pol}} \times \mathrm{B}_{z}$ force, which restores force balance again.

Fig. 2. The toroidal magnetic field must increase beyond the edge of the plasma in order to conserve toroidal flux within the toroidal field coils as the diamagnetism in the center of the plasma is increased. The additional toroidal field may be supported by force-free currents (1) or it may be a vacuum field (2).

Fig. 3. The outward radial velocity produced by raising the plasma pressure also induces a longitudinal $\mathrm{v}_{\mathrm{r}} \times \mathrm{B}_{\mathrm{pol}}$ component that results in a decrease of the poloidal magnetic field. This process does not restore force balance at a higher plasma pressure.

Fig. 4. The q profile as a function of radius is convected as the plasma at the edge is compressed against a flux conserving wall.

Fig. 5. Dynamics of a flux conserving plasma (shown schematically) in a continuously driven system with energy losses at the edge of the plasma. 


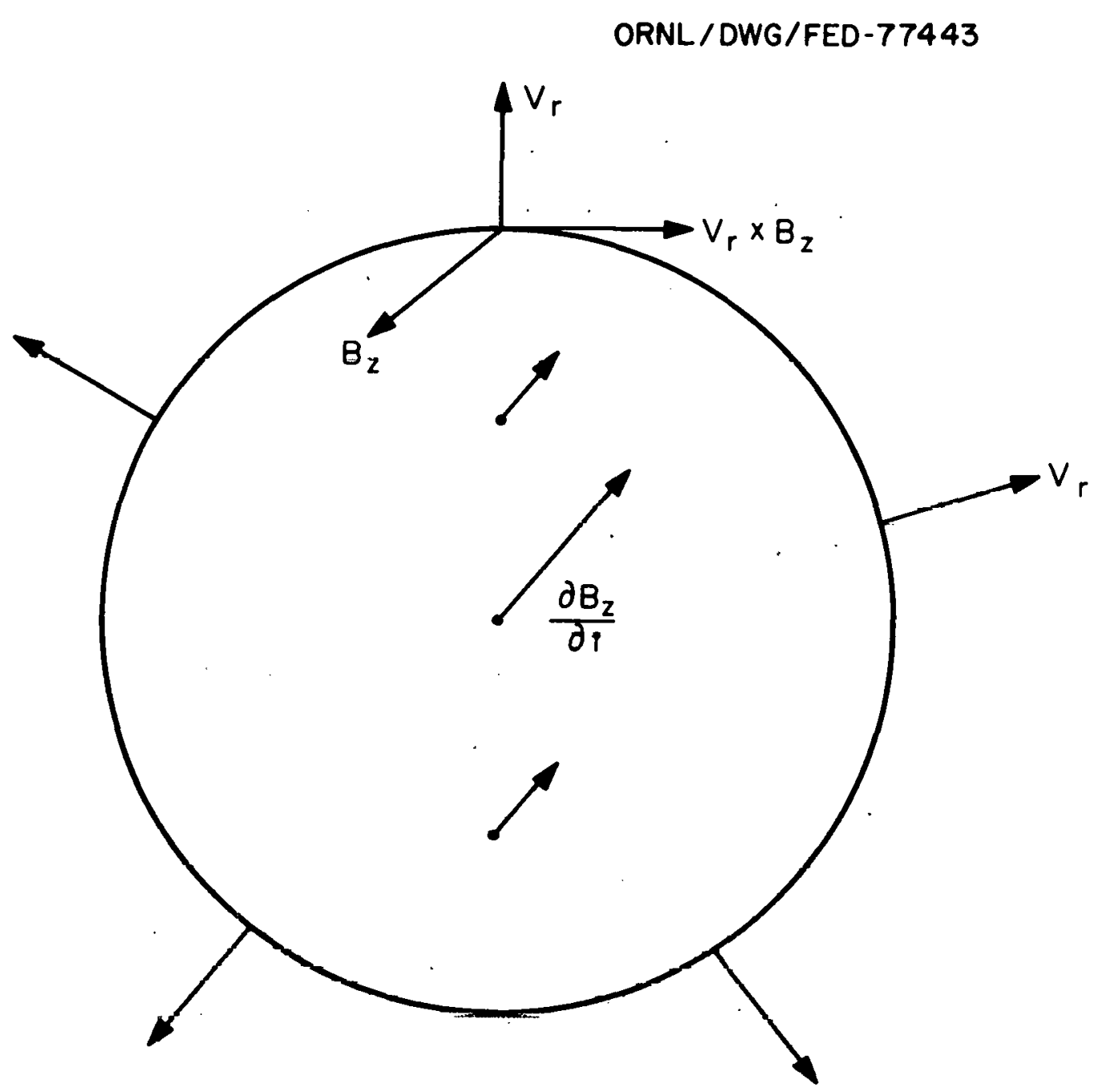

Tig. 1. 


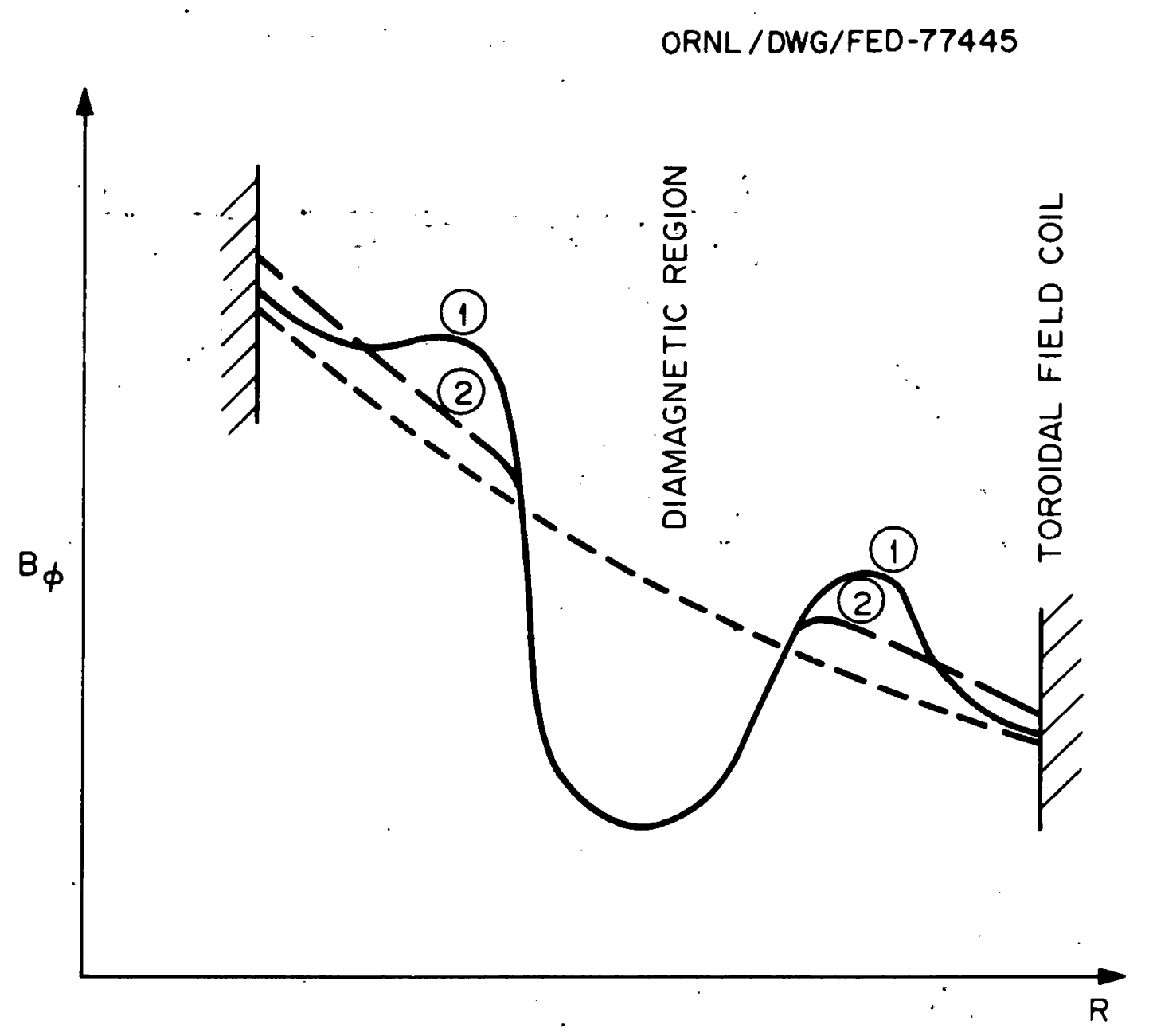

Fig. 2. 
ORNL/DWG/FED-77442

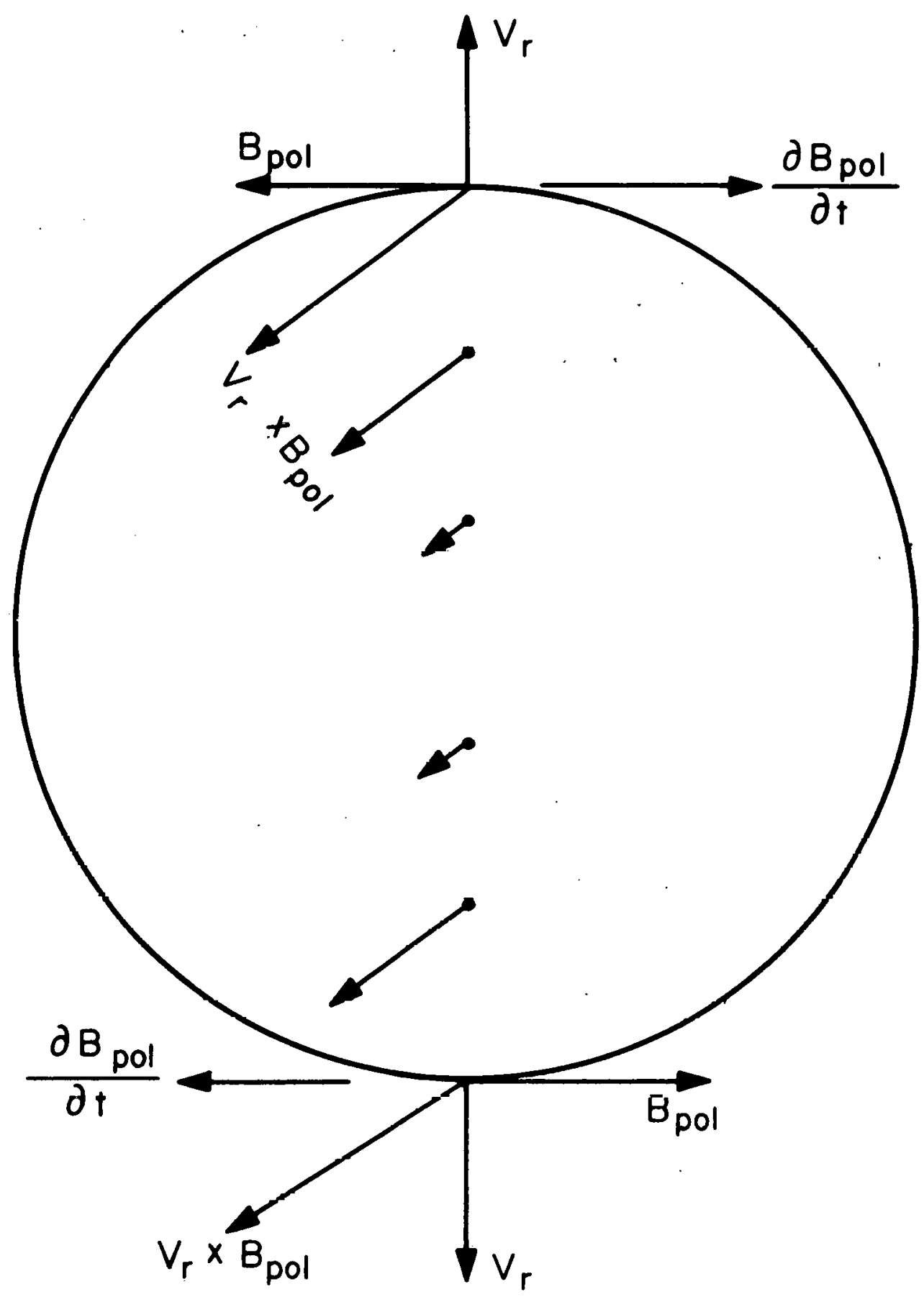

Fig. 3. 
ORNL /DWG/FED-77444

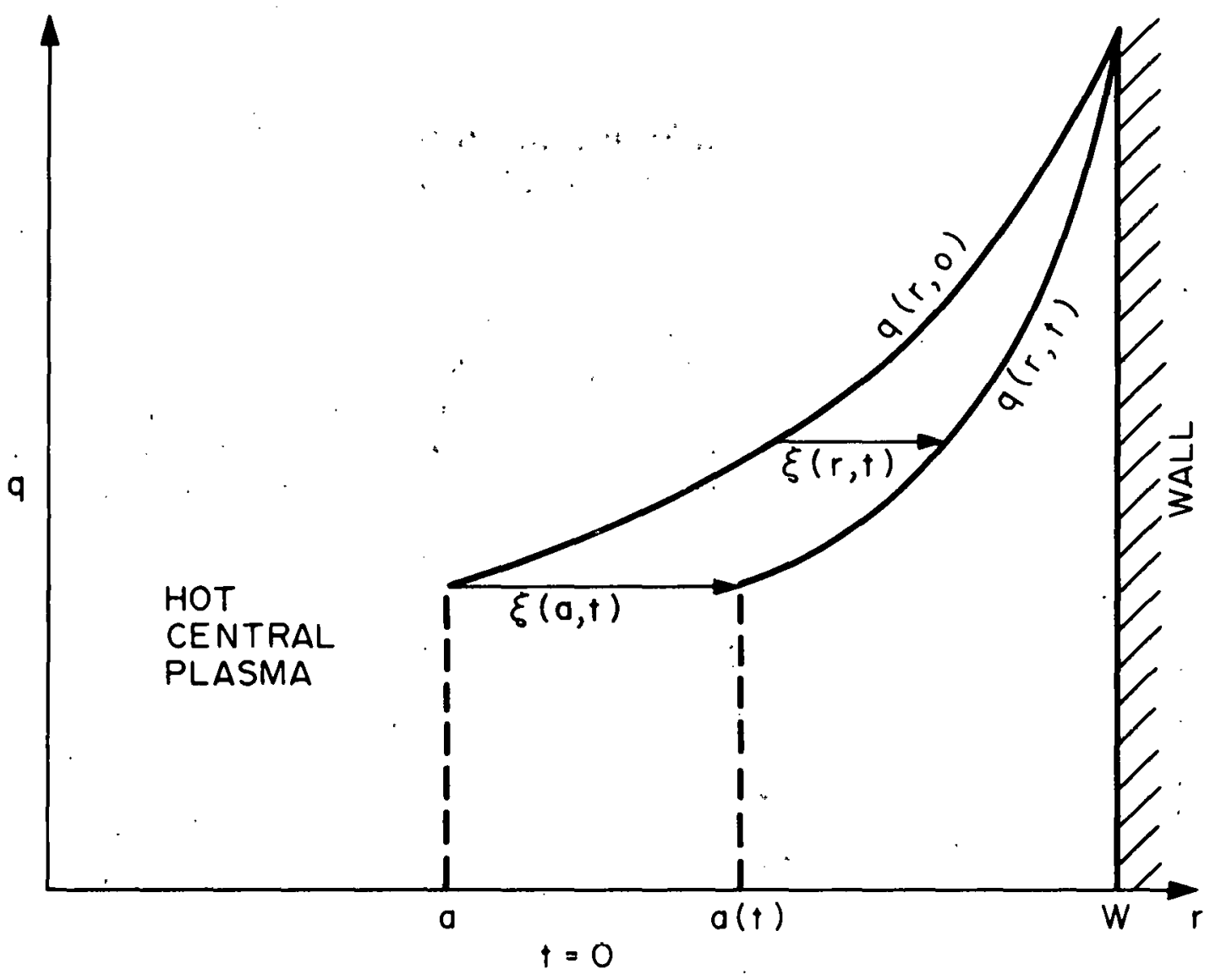

Fig. 4. 
ORNL / DWG/FED-77446

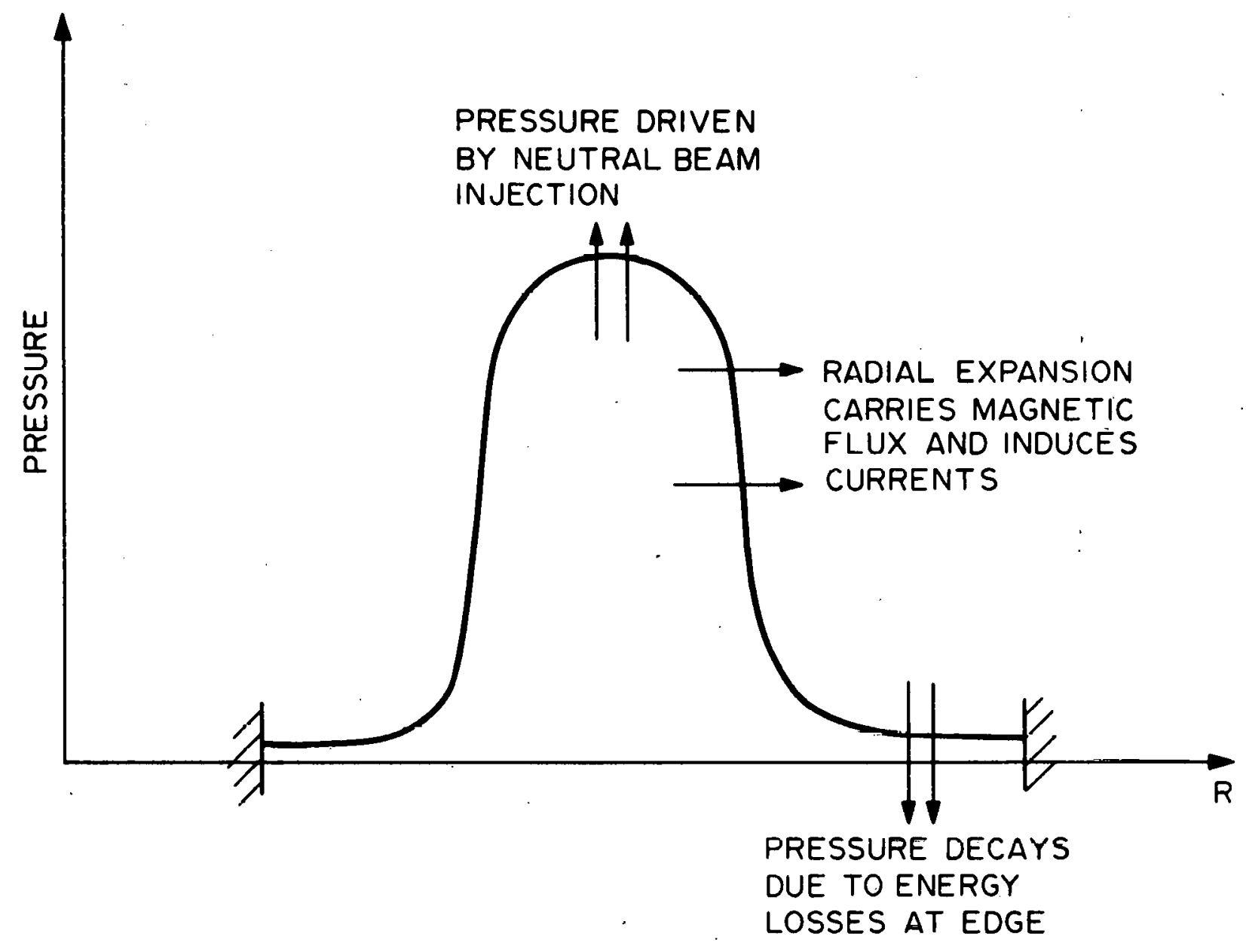

Fig. 5. 


\author{
ORNL/TM-6271 \\ Dist. Category UC-20 g
}

\title{
INTERNAL DISTRIBUTION
}

\author{
1. L. A. Berry \\ 2. J. D. Callen \\ 3. R. A. Dand1 \\ 4. R. A. Dory \\ 5. G. G. Kelley \\ 6. H. H. Haselton \\ 7. P. N. Haubenreich \\ 8. M. S. LubelI \\ 9. 0. B. Morgan \\ 10. H. Postma \\ 11. M. W. Rosenthal
}

\author{
12. J. Sheffield \\ 13. D. Steiner \\ 14-47. Glenn Bateman \\ 48-49. Laboratory Records Department \\ 50. Laboratory Records, ORNL-RC \\ 51. Document Reference Section \\ 52-53. Central Research Library \\ 54. Fusion Energy Division Library \\ 55. Fusion Energy Division \\ Communications Center \\ 56. ORNL Patent Office
}

\section{EXTERNAL DISTRIBUTION}

57. Bibliothek, Max-Planck Institute für Plasmaphysik, 8046 Garching bei Minchen, Federal Republic of Germany

58. Bibliothèque, Service du Confinement des Plasmas, C.E.A., B.P. No. 6, 92, Fontenay-aux Roses (Seine), France

59. Lung Cheung, Department of Electronics, University Science Center, The Chinese University of Hong Kong, Shatin, N.T., Hong Kong

60. J. F. Clarke, Office of Fusion Energy, G-234, Department of Energy, Washington, DC 20545

61. R. W. Conn, Fusion Technology Program, Nuclear Engineering Department, University of Wisconsin, Madison, WI 53706

62. CTR Library, c/o Alan F. Haught, United Technologies Research Laboratory, East Hartford, CT 06108

63. CTR Reading Room, c/o Allan N. Kaufman, Physics Department, University of California, Berkeley, CA 94720

64. J. Nar1 Davidson, School of Nuclear Engineering, Georgia Institute of Technology, Atlanta, GA 30332

65. Documentation S.I.G.N., Départment de la Physique du Plasma et de la Fusion Controlée, Association EURATOM-CEA sur la Fusion, Centre d'Études Nucléaires, B'.P. 85, Centre du TRI, 38041 Grenoble, Cedex, France

66. W. R. Ellis, Office of Fusion Energy, G-234, Department of Energy, Washingcon, DC 20545

67. Harold K. Forsen, Exxon Nuclear Co., Inc., 777 106th Avenue, N.E., C-000777, Bellevue, WA 98009

68. Harold P. Furth, Princeton Plasma Physics Laboratory, Princeton University, Forrestal Campus, P.0. Box 451, Princeton, NJ 08540

69. Roy W. Gould, California Institute of Technology, Mail Stop 116-81, Pasadena, CA 91125

70. Robert L. Hirsch, Exxon Research and Engineering, P.0. Box 101, Florham Park, NJ 07932 
71. Raymond A. Huse, Manager, Research and Development, Public Service Gas and Electrlc Company, 80 Park Place, Newark, NJ 07101

72. T. Hsu, Office of Fusion Energy, G-234, Department of Energy, Washington, DC 20545

73. V. E. Ivanov, Physical-Technical Institute of the Ukranian Academy of Sciences, Sukhumi, U.S.S.R.

74. A. Kadish, Office of Fusion Energy, G-234, Department of Energy, Washington, DC 20545

75. L. M. Kovrizhnikh, Lebedev Institute of Physics, Academy of Sciences of the U.S.S.R., Leninsky Prospect 53, Moscow, U.S.S.R.

76. Guy Lava1, Groupe de Physique l'héorique, Ecole Polytechnique, 91 Pa1nispall, Partis, France

77. Library, Centre de Recherches en Physique des Plasma, 21 Avenue des Bains, 1007, Lausanne, Switzerland

78. Library, Culham Laboratory, United Kingdom Atomic Energy Authority, Abingdon, Oxon, OX14 3DB, United Kingdom

79. Libraxy, FOM-Institut voor Plasma - Fysica, Rijnhuizen, Jutphaas, Netherlands

80. Library, Inst1tute for Plasma Physics, Nagoya University, Nagoya, Japan 464

81. Library, International Centre for Theoretical Physics, Trieste, Italy

82. Library, Laboratorio Gas Ionizzati, Frascati, Italy

83. Dsumber G. Lominadze, Academy of Sciences of the Georgian S.S.R., 8 Dzerzhinski St., 38004, Tbilisi, U.S.S.R.

84. Oscar P. Manley, Offlce of Fuston Energy, G-234, Department of Energy, Washington, DC 20545

85. D. G. McAlees, Exxon Nuclear Co., Inc., Research and Technology Laser Enrichment Department, 2955 George Washington Way, Richland, WA 99352

86. J. E. McCune, School of Engineering, Department of Aeronautics and Astronautics, Bldg. 37-391, Massachusetts Inst1tute of Technology, Cambridge, MA 02139

87. Claude Mercier, Service du Theorie des Plasmas, Centre d'Études Nuclêaires, Fontenay-aux-Roses (Seine), France

88. K. G. Moses, Office of Fusion Energy, G-234, Department of Energy, Washington, DC 20545

89. D. Pfirsch, Institute for Plasma Physics, 8046 Garching bei München, Federal Republic of Germany

90. Plasma Physics Group, Department of Engineering Physics, Australian National University, P.O. Box 4, Canberra A.C.T. 2600, Australia

91. Robert E. Price, Office of Fusion Energy, G-234, Department of Energy, Washington, DC 20545

92. A. Rogister, Inetitute for Plasma Physics, KFA, Fostfach 1913, D-5170, Jilich 1, Federal Republic of Germany

93. W. Sadowski, Office of Fusion Energy, G-234, Department of Energy, Washington, DC 20545

94. V. D. Shafranov, I. V. Kurchatov Institute of Atomic Energy, 46 Ulitsa Kurchatova, P.O. Box 3402, Moṣcow, U.S.S.R. 
37

95. Xu. S. Sigov, Institute of Applied Mathematics of the U.S.S.R. Academy of Sciences, Miuskaya, Sq. 4, Moscow A-47, U.S.S.R.

96. W. M. Stacey, Jr., School of Nuclear Engineering, Georgia Institute of Technology, Atlanta, GA 30332

97. J. B. Taylor, Culham Laboratory, United Kingdom Atomic Energy Authority, Abingdon, Oxon, 0X14 3DB, United Kingdom

98. Thermonuclear Library, Japan Atomic Energy Research Institute, Toka1, Naka, Ibarak1, Japan

99. Francisco Verdaguer, Director, Division of Fusion, Junta de Energia Nuclear, Madrid 3, Spain

100. Director, Research and Technical Support Division, Department of Energy, Oak Ridge Operations, P.O. Box E, Oak Ridge, TN 37830

101-276. Given distribution as shown in TID-4500, Magnetic Fusion Energy

$\frac{\frac{101}{175}}{176}$ (Distribution Category UC-20g, Theoretical Plasma Physics) 